\title{
Planarer Vierleiter-Transducer für impedanzspektroskopische Material- und Sensorcharakterisierung
}

\author{
Gunter Hagen, Jaroslaw Kita, Daniela Schönauer-Kamin, Ralf Moos \\ Lehrstuhl für Funktionsmaterialien, Universität Bayreuth, 95440 Bayreuth, Deutschland \\ gunter.hagen@uni-bayreuth.de
}

\begin{abstract}
Zusammenfassung
Die Bestimmung der elektrischen Eigenschaften von Funktionsmaterialien in Sensoranwendungen stellt oftmals eine Herausforderung dar. Impedanzspektroskopische Untersuchungen geben Aufschluss über Leitfähigkeitsmechanismen oder dielektrische Eigenschaften der beteiligten Materialien. Allerdings sind dabei oftmals Elektroden- oder Grenzflächeneffekte beteiligt, die einen Rückschluss auf reine Materialgrößen erschweren. Genauere Ergebnisse zur Charakterisierung bietet die Vierleiter-Impedanzspektroskopie, die allerdings zumeist an kompakten Materialproben angewendet wird. Im vorliegenden Beitrag wird ein Sensor-Transducer vorgeschlagen, der die Anwendung solcher Messungen rein planar, also auch bei Sensorschichten oder in Flüssigkeiten ermöglicht. Geometrieunabhängige Materialkennwerte können durch Einführung eines Geometriefaktors erhalten werden. Der Aufbau des Transducers in keramischer Mikrosystemtechnik erlaubt außerdem die Integration eines Heizelements und damit die schnelle und unkomplizierte Anwendung der Vierleiter-Impedanzspektroskopie in sensornahen Strukturen auch bei erhöhter Temperaur.
\end{abstract}

Key words: Vierleiter-Impedanzspektroskopie, Dielektrizitätszahl, Leitfähigkeit, Interdigitalelektroden

\section{Motivation}

Die Impedanzspektroskopie ist ein geeignetes Werkzeug zur elektrischen oder elektrochemischen Charakterisierung unbekannter Materialien oder Flüssigkeiten. Häufig spielen aber dabei auch Effekte oder Reaktionen an den kontaktierenden Elektroden eine wichtige Rolle.

Bei der klassischen Zweileiter-Impedanzspektroskopie gehen alle die Impedanz bestimmenden Mechanismen zwischen den Anschlusskontakten in die Messung mit ein. In einem weiten Frequenzbereich wird die Antwort des Systems (bestehend aus Zuleitungen, Elektroden, Grenzflächen und dem zu untersuchenden Material) auf eine Wechselspannungsanregung aufgenommen und als komplexer Widerstand dargestellt. Im Idealfall können dann die frequenzabhängig unterschiedlichen Beiträge voneinander getrennt und Mechanismen im Material zugeordnet werden.

Um den reinen Materialanteil separiert von Elektroden- oder Grenzschichteffekten auch messtechnisch direkt erfassen zu können, ist eine Vierleiter-Messung sinnvoll [1]. Dazu wird das Material mit zwei zusätzlichen Elektroden zwischen den Anregungselektroden kontaktiert, um die Antwort des Systems separat und damit stromlos messen zu können. Im Bereich der Sensorik beziehen sich solche Fragestellungen zumeist auf planare Anordnungen bzw. dünne Schichten.

Im vorliegenden Fall wurde deshalb ein vollständig planarer Aufbau gewählt. Die Elektroden sind dabei als Interdigital-Elektroden zur Anregung des Systems mit dazwischen laufenden mäandrierenden Elektroden zur Messung der Systemantwort ausgeführt (Bild 1).

Eine solche Anordnung (Elektroden in Dünnschichttechnik) konnte bereits erfolgreich zur Vermessung ionenleitender ZeolithDickschichten verwendet werden. In diesem Fall wurde der Funktionsmechanismus eines Kohlenwasserstoff-Sensors, der an der Grenzschicht zwischen dem Zeolith-Material und einem damit in Kontakt stehenden Metalloxid untersucht [2].

In einem zweiten Schritt wurde dann die planare Elektrodenstruktur in Dickschichttechnik auf ein Sensorbauteil mit integriertem Heizelement übertragen [3]. Mit diesem Aufbau können nun schnell und unkompliziert beliebige 
Materialien (in Form einer Beschichtung) oder Flüssigkeiten charakterisiert werden. Der Einfluss von Sensorelektroden bzw. ihr Einfluss auf die Leitfähigkeitseigenschaften und / oder -mechanismen kann ermittelt werden.

Für die Auswertung geometrieunabhängiger Materialeigenschaften (wie Dielektrizitätszahl oder Leitfähigkeit) muss der besondere Feldverlauf zwischen den planaren interdigitalen Elektroden berücksichtigt werden.

Zu diesem Zweck wurde eine Kalibrierung des Transducers mit einem bekannten Material (hier Wasser) durchgeführt und ein Geometriefaktor ermittelt, der die Kapazitätsberechnung auf einen einfachen Plattenkondensator-Fall zurückführt.

Um die Kapazitätswerte exakt ermitteln zu können ist außerdem die Kenntnis der Leerkapazität des Sensors ohne Zuleitungseinflüsse nötig.

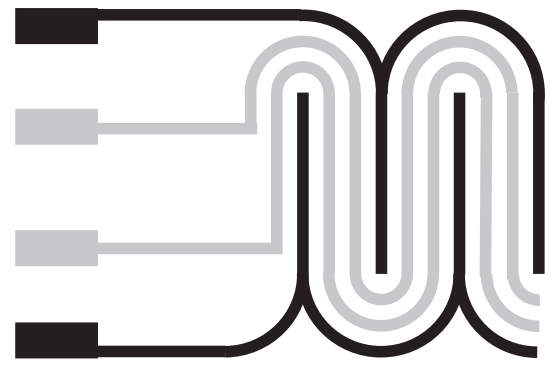

Bild 1. Schematische Darstellung der Anordnung planarer Vierleiter-Elektroden mit Zuleitungen.

\section{Experimentelles}

Die Herstellung des Sensorträgers erfolgte durch Siebdruck von Elektrodenmaterial (z.B. $\mathrm{Au}$ oder $\mathrm{Pt}$ ) auf keramischen Substraten. Dabei werden die vier Zuleitungen und eine am Ende der Zuleitungen liegende flächige Beschichtung hergestellt. Die Strukturierung der interdigitalen Vierleiter-Elektroden erfolgt nachträglich mittels eines Lasers (LPKF Microline). Damit lassen sich bzgl. der Elektrodenfinger Linienbreiten und -abstände von ca. $30 \mu \mathrm{m}$ erreichen. Messergebnisse im vorliegenden Beitrag beziehen sich auf einen Aufbau mit einer laserstrukturierten Au-Elektrodenstruktur auf einem keramischen $\mathrm{Al}_{2} \mathrm{O}_{3}$-Substrat (Bild 2a). Der Anschluss des Sensors erfolgte mittels angelöteten Litzen. Impedanzspektroskopische Messungen wurden mit einem Alpha-Analyzer (Novocontrol) im Frequenzbereich von $100 \mathrm{~Hz}$ bis $1 \mathrm{MHz}$ durchgeführt.

\section{Korrektur der Leerkapazität}

Die Kenntnis der reinen Leerkapazität der Elektrodenstruktur ist nötig, um bei der Angabe von Materialkennwerten frei von Zuleitungseinflüssen $\mathrm{zu}$ sein. Es wurden verschiedene Messungen an einem unbeschichteten Sensor (Bild 2a) und an den Zuleitungen ohne Kontakt zur Elektrodenstruktur (Bild 2c) durchgeführt. Die Subtraktion der (gemessenen) komplexen Admittanzen $\underline{Y}_{\text {Leer }}$ und $\underline{Y}_{\text {zuleitung }}$ führt damit zu einer korrigierten Leerkapazität $C_{\text {Leer,korr }}$ der Struktur.

a)

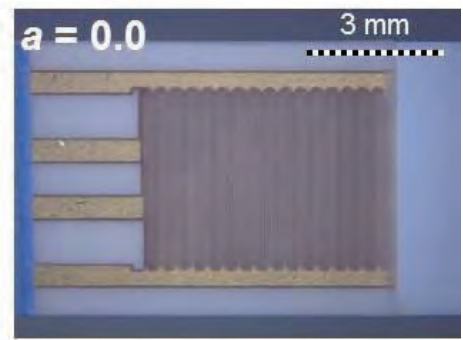

b)

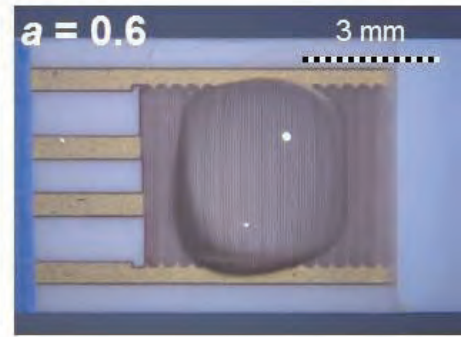

c)

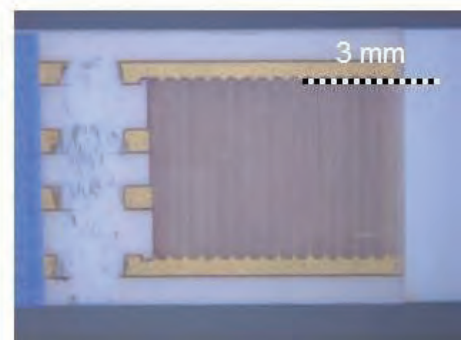

Bild 2. Vierleiter-Transducer im Bereich der laserstrukturierten Elektroden: a) ohne Beschichtung, b) mit $60 \%$-iger Wasser-Bedeckung, c) mit getrennten Zuleitungen zur Vermessung der reinen Zuleitungseinflüsse. Abgeändert nach [3] mit Genehmigung von Elsevier. 


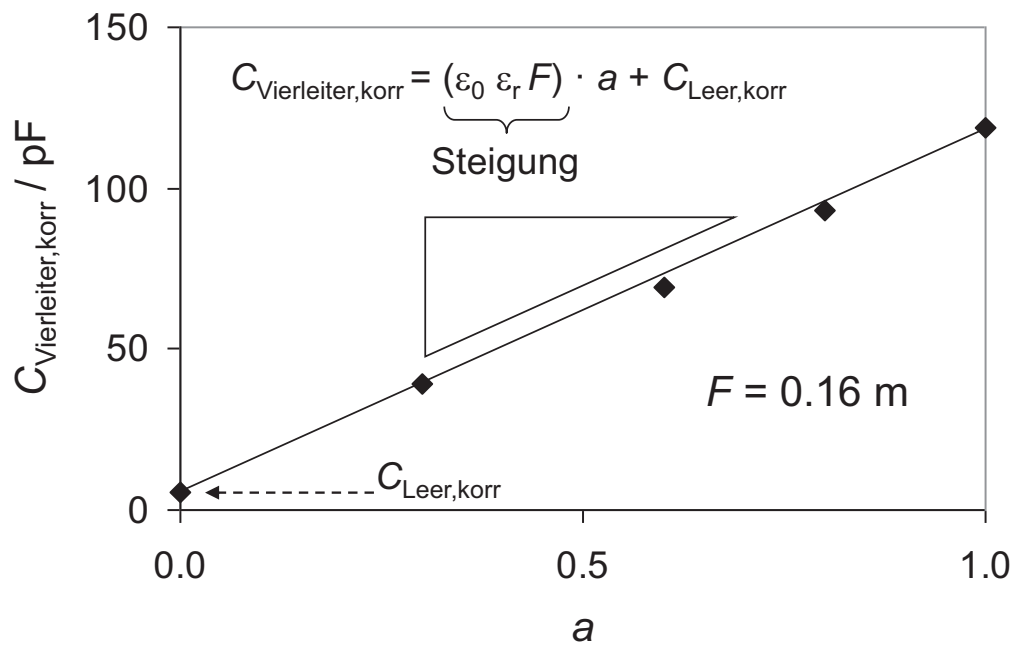

Bild 3. Ermittlung des Geometriefaktors F für die vorliegende Struktur. Der Bedeckungsgrad mit Wasser, a, ist auf der Abszisse aufgetragen.

\section{Kalibrierung bzgl. Substrateinfluss und Feldlinienverlauf}

Um bei Testmaterialien auf der planaren Anordnung Materialeigenschaften wie die Dielektrizitätszahl oder die Leitfähigkeit quantitativ bestimmen zu können, wurde eine Kalibrierung des Sensors durchgeführt.

Messungen an einem Sensor mit verschiedener Wasser-Bedeckung (Bedeckungsgrad $a, \varepsilon_{\mathrm{r}}=$ 81, siehe z.B. Bild 2b) zeigen einen linearen Anstieg der um die Zuleitungseinflüsse korrigierten Kapazität $C_{\text {Vierleiter,korr }}$ über steigender Wasser-Bedeckung (Bild 3).

Über die Steigung lässt sich ein speziell für diesen Aufbau gültiger Geometriefaktor $F$ errechnen, der die Bestimmung der Kapazität oder des Widerstandes auf die typische Geometrie eines Plattenkondensators mit homogener Feldverteilung zurückführt und den Substrateinfluss sowie die Elektrodengeometrie beinhaltet. Dabei gilt Gleichung (1):

$F=\left(C_{\text {Vierleiter,korr }}-C_{\text {Leer,korr }}\right) /\left(\varepsilon_{0} \cdot \varepsilon_{\mathrm{r}} \cdot a\right)$

Der Geometriefaktor kann nun bei Vermessung unbekannter Materialien zur Berechnung geometrieunabhängiger Materialkennwerte herangezogen werden.

Die Leitfähigkeit wird demnach aus der Auswertung der Impedanzspektren der Vierleiter-Messung ermittelt. Dabei ist der ohmsche Widerstands-Anteil des Bulk-Materials $(R)$ meist direkt aus der Nyquist-Ortskurve ablesbar, da Elektrodenimpedanzen nicht in das Messergebnis einfließen. Für die Leitfähigkeit gilt dann Gleichung 2.

$\rho=1 / \sigma=R \cdot F$
Diese Berechnung kann man jedoch nur für den Fall anwenden, dass alle elektrischen Feldlinien im Testmaterial verlaufen, was durch eine ausreichende Beschichtungsdicke gewährleistet wird [3,4]. Dazu muss die Beschichtungsdicke wesentlich größer als der Elektrodenabstand (hier also ca. $30 \mu \mathrm{m}$ ) sein. Für die Anwendung des Transducers zur Charakterisierung dünner Schichten muss die Berechnung auf andere Weise erfolgen.

\section{Zusammenfassung}

Mit dem vorliegenden Transducer ist es möglich, Materialien in Form einer Beschichtung oder auch Flüssigkeiten elektrisch zu charakterisieren ohne dabei von den Einflüssen der kontaktierenden Elektroden oder dadurch entstehender Grenzschichteffekte beeinflusst zu sein.

Einflüsse auf die Kapazitätsmessung, die durch die Zuleitungen hervorgerufen werden, konnten korrigiert werden.

Um bei der Vermessung unbekannter Materialien geometrieunabhängige Größen wie Dielektrizitätszahl oder Leitfähigkeit angeben zu können, muss der besondere Feldlinienverlauf bei der planaren Anordnung berücksichtigt werden. Ein ermittelter Geometriefaktor $F$ für die vorliegende Struktur führt die Kapazitätsberechnung auf den typischen Fall eines Plattenkondensators zurück.

Ein rückseitig aufgebrachter Dickschicht-Heizer ermöglicht weiterhin, bei definierten Temperaturen zu messen.

\section{Ausblick}

In weiteren Arbeiten soll das System auch theoretisch betrachtet werden. Simulationen 
des Feldverlaufs bei der Vierleiter-Messung sollen den Geometriefaktor allgemein beschreiben und das Verständnis von Einflüssen auf die Qualität der ermittelten Werte vertiefen.

\section{Literatur}

[1] E. Barsoukov, J.R. Macdonald, Impedance Spectroscopy, 2nd ed., John Wiley \& Sons, Hoboken, NJ, 2005.

[2] G. Hagen, A. Schulz, M. Knörr, R. Moos, FourWire Impedance Spectroscopy on Planar Zeolite/Chromium Oxide Based Hydrocarbon Gas Sensors, Sensors, 2007, 7, 2681-2692, doi: doi:10.3390/s7112681

[3] G. Hagen, J. Kita, N. Izu, U. Röder-Roith, D. Schönauer-Kamin, R. Moos, Planar platform for temperature dependent four-wire impedance spectroscopy - novel tool to characterize functional materials, Sensors and Actuators B: Chemical, 2013, 187, 174-183, doi: 10.1016/j.snb.2012.10.068

[4] G. Hagen, Impedimetrische Gassensoren auf Zeolith-Basis, Dissertationsschrift, Shaker-Verlag, Aachen, 2009 